ARTICLE

\title{
A comprehensive influenza reporter virus panel for high-throughput deep profiling of neutralizing antibodies
}

Adrian Creanga ${ }^{1}$, Rebecca A. Gillespie ${ }^{1}$, Brian E. Fisher ${ }^{1}$, Sarah F. Andrews ${ }^{1}$, Julia Lederhofer (1) ${ }^{1}$, Christina Yap ${ }^{1}$, Liam Hatch', Tyler Stephens², Yaroslav Tsybovsky², Michelle C. Crank, Julie E. Ledgerwood', Adrian B. McDermott (i) ${ }^{1}$, John R. Mascola ${ }^{1}$, Barney S. Graham (iD) ${ }^{1 凶} \&$ Masaru Kanekiyo (iD ${ }^{1 凶}$

Broadly neutralizing antibodies (bnAbs) have been developed as potential countermeasures for seasonal and pandemic influenza. Deep characterization of these bnAbs and polyclonal sera provides pivotal understanding for influenza immunity and informs effective vaccine design. However, conventional virus neutralization assays require high-containment laboratories and are difficult to standardize and roboticize. Here, we build a panel of engineered influenza viruses carrying a reporter gene to replace an essential viral gene, and develop an assay using the panel for in-depth profiling of neutralizing antibodies. Replication of these viruses is restricted to cells expressing the missing viral gene, allowing it to be manipulated in a biosafety level 2 environment. We generate the neutralization profile of 24 bnAbs using a 55-virus panel encompassing the near-complete diversity of human H1N1 and H3N2, as well as pandemic subtype viruses. Our system offers in-depth profiling of influenza immunity, including the antibodies against the hemagglutinin stem, a major target of universal influenza vaccines.

\footnotetext{
${ }^{1}$ Vaccine Research Center, National Institute of Allergy and Infectious Diseases, National Institutes of Health, Bethesda, MD, USA. ${ }^{2}$ Electron Microscopy Laboratory, Cancer Research Technology Program, Frederick National Laboratory for Cancer Research sponsored by the National Cancer Institute, Frederick,

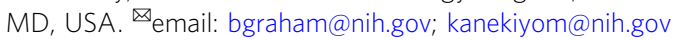


nfluenza virus continues to cause seasonal epidemics and pandemics despite vaccine availability. In addition, highly pathogenic avian influenza viruses cause sporadic outbreaks in humans with a high mortality rate, posing potential risk of human adaptation and are considered pandemic threats ${ }^{1}$. Viral glycoprotein hemagglutinin (HA) is required for viral entry into the host cell through binding to sialic acid moieties on glycoproteins or glycolipids and mediates fusion of the viral membrane with host endosomal membranes. Another viral glycoprotein neuraminidase (NA) cleaves sialic acid and promotes the release of progeny viruses from infected cells. Neutralizing antibodies are primarily directed against $\mathrm{HA}$ and can compete for receptor binding, inhibit the membrane fusion machinery, or cause virus aggregation. Antibodies to NA can sometimes have neutralizing activity but are thought to primarily limit virus egress by inhibiting enzymatic activity and release of virus thereby inhibiting viral spread. Based on the genetic and antigenic properties of $\mathrm{HA}$ and NA, influenza A viruses are divided into groups 1 and 2, each of which have several subtypes defined primarily by HA. To date, there are $18 \mathrm{HA}$ and $11 \mathrm{NA}$ subtypes identified and characterized $^{2}$. Currently, strains of $\mathrm{H} 1 \mathrm{~N} 1$ and $\mathrm{H} 3 \mathrm{~N} 2$ influenza $\mathrm{A}$ as well as influenza $\mathrm{B}$ viruses co-circulate in humans. In addition, several other subtypes of animal influenza viruses (e.g., H5N1, H6N1, H7N9, H9N2, and H10N8) can also infect humans and occasionally result in mortality. In contrast to influenza A viruses, which have an extensive zoonotic reservoir, influenza $B$ viruses are isolated almost exclusively from humans with a more limited evolutionary history and have diverged into only two genetically and antigenically distinct lineages (Victoria- and Yamagata-like lineages) ${ }^{3}$.

The discovery of broadly neutralizing antibodies (bnAbs) capable of neutralizing multiple influenza virus subtypes in humans opens an opportunity for developing a universal influenza vaccine, which elicits such antibodies ${ }^{4-6}$. Many of these antibodies target conserved epitopes in the HA stem and neutralize virus by inhibiting the viral fusion machinery, therefore, the activity is not detectable by traditional hemagglutination inhibition (HAI) assay, which measures the ability of antibody to inhibit virus-receptor interaction. Several less broad bnAbs recognize the receptor-binding site (RBS) in the HA head, hence exhibiting HAI activity ${ }^{7}$. Since most universal influenza vaccine candidates aim to induce protective levels of such bnAb response $^{6,8}$, comprehensive analysis of the neutralization breadth and potency regardless of HAI activity is crucial to accelerate the efforts to develop effective universal influenza vaccines. The influenza microneutralization $(\mathrm{MN})$ assay has been the most commonly used method to measure neutralizing activity of antibodies against influenza virus ${ }^{9}$. In this assay, virus replication is measured by either detecting viral nucleoprotein (NP) with an enzyme-linked immunosorbent assay (ELISA), titrating hemagglutination, or scoring cytopathic effects. Plaque-reduction neutralization assay is also commonly used for influenza and other viruses. These approaches are labor-intensive, not easily scalable, and the handling of live viruses of animal origin requires highcontainment laboratories. There is also significant performance variability between laboratories due to multi-step signal amplification or reliance on subjective scoring. Given these inevitable limitations of the current $\mathrm{MN}$ assay, there is a need to transform the $\mathrm{MN}$ assay to be safe, high throughput, robust, easy to standardize, and automation compatible ${ }^{10}$.

The use of reporter viruses for developing standardized highthroughput neutralization assays has greatly advanced our ability to measure and characterize antibody responses induced by infection and/or vaccination ${ }^{11-13}$. While HA/NA pseudotyped reporter lentiviruses have been constructed for influenza and utilized effectively for measuring neutralizing activity ${ }^{14}$, they are often criticized for not recapitulating some of the key aspects of influenza viruses, such as viral morphology and HA spike density ${ }^{15,16}$. Therefore, building influenza viruses with a reporter feature is an attractive alternative. Among several approaches to produce influenza reporter viruses, a replication-competent reporter virus can be developed by fusing a reporter gene to a viral gene (e.g., $\mathrm{PA}$ or $\mathrm{NS})^{17}$. This new virus remains as virulent as the parental virus in animals, making it suitable for viral pathogenesis studies, yet still subject to biosafety precautions. In contrast, single-cycle infectious or replication-restricted reporter (R3) influenza viruses can be generated by replacing one of the viral essential genes (e.g., PB1 or HA) with a reporter gene ${ }^{17-20}$. Thereby these R3 viruses are capable of replicating only in cells complementing the deleted viral gene product in trans, making it safe to handle in low-containment laboratories.

In the present study, we developed a comprehensive panel of R3 viruses to enable high-throughput and in-depth influenza virus neutralization profiling. We generated a neutralization matrix of 24 monoclonal antibodies (mAbs) and $55 \mathrm{R} 3$ viruses spanning 6 subtypes of influenza virus and compared the R3 virus-based assay with the gold-standard ELISA-based MN assay ${ }^{9}$. The reporter virus assay provides a more robust method for probing the breadth of anti-influenza immunity needed for developing universal influenza vaccines.

\section{Results}

Generation of R3 $\mathbf{P B B} 1$ viruses. The influenza virus has a segmented genome, which allows its rapid and relatively easy genetic manipulation. Each segment consists of either one or two protein-coding sequences flanked by short noncoding regions (NCRs). The genome packaging signal sequences are located at both $3^{\prime}$ - and $5^{\prime}$-termini of each segment encompassing the entire NCRs and part of the adjacent protein-coding sequence. Genome packaging sequences are unique to each segment and required for an efficient incorporation of each viral RNA molecule into the virion. The PB1 segment encodes two proteins: PB1, the main component of the viral RNA-dependent RNA polymerase, and $\mathrm{PB} 1-\mathrm{F} 2$, translated from the +2 frame and not required for virus replication ${ }^{21}$. The protein-coding sequence of PB1 (A/WSN/1933, $\mathrm{H} 1 \mathrm{~N} 1$ ) has 2,274 nucleotides and is flanked by 24 bases at the $3^{\prime}$ and 43 bases at $5^{\prime}$-termini. In addition to the NCRs, genome packaging signals of the PB1 segment comprise 120 bases of the coding region at both $3^{\prime}$ - and $5^{\prime}$-termini ${ }^{18,22}$ (Supplementary Fig. 1).

To build R3 influenza viruses, we altered the PB1 segment by removing the $\mathrm{PB} 1$ coding sequence not required for genome packaging and replacing it with the reporter encoding fluorescent protein tdKatushka2 ${ }^{23}$. To prevent translation of alternative transcripts from PB1 segments, we mutated potential initiation codons (ATGs) found between the $3^{\prime}$ end and the reporter open reading frame (ORF) (Fig. 1a). PB1 is essential for virus replication therefore, $\mathrm{R} 3$ influenza viruses in which $\mathrm{PB} 1$ was removed $(\mathrm{R} 3 \triangle \mathrm{PB} 1)$ can be propagated only in cells expressing the PB1 in trans (Fig. 1b, c). Thus, we used MDCK-SIAT1 cells, which constitutively express human $\beta$-galactoside $\alpha 2,6$-sialyltransferase 1 (SIAT1) ${ }^{24}$ to prepare a cell line constitutively expressing $\mathrm{PB} 1$ of A/WSN/1933 by transfecting a plasmid encoding a puromycin-resistance gene, $\mathrm{PB} 1$, and a self-cleaving peptide derived from Thosea asigna virus 2A (T2A) in between the two genes. The $\mathrm{R} 3 \triangle \mathrm{PB} 1$ virus was rescued by reverse genetics using 8 plasmids encoding $\mathrm{HA}$ and NA of wild-type $\mathrm{H} 1 \mathrm{~N} 1$ or H3N2 influenza A viruses; PB2, PA, NP, M, and NS of A/WSN/ 1933; and another encoding the reporter. To validate the use of $\mathrm{R} 3 \triangle \mathrm{PB} 1$ viruses for influenza neutralization assays, we also rescued replication-competent $\mathrm{H} 1 \mathrm{~N} 1$ and $\mathrm{H} 3 \mathrm{~N} 2$ parental viruses, 


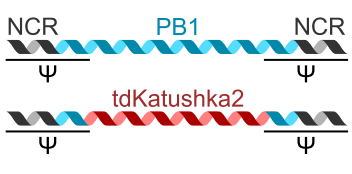

b
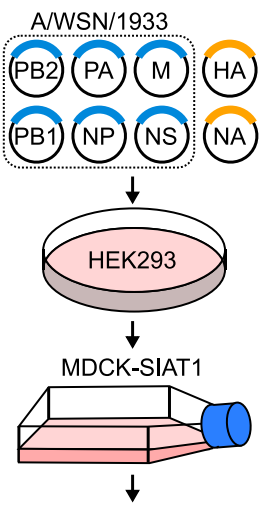

Parental virus

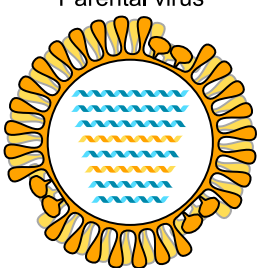

Resultant virus with desired HA/NA

d

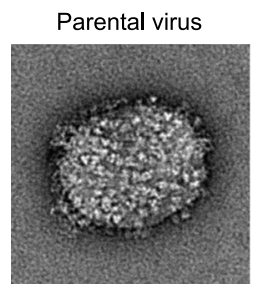

Reverse genetics plasmids

Rescue

Propagation

$\mathrm{R} 3 \triangle \mathrm{PB} 1$ virus
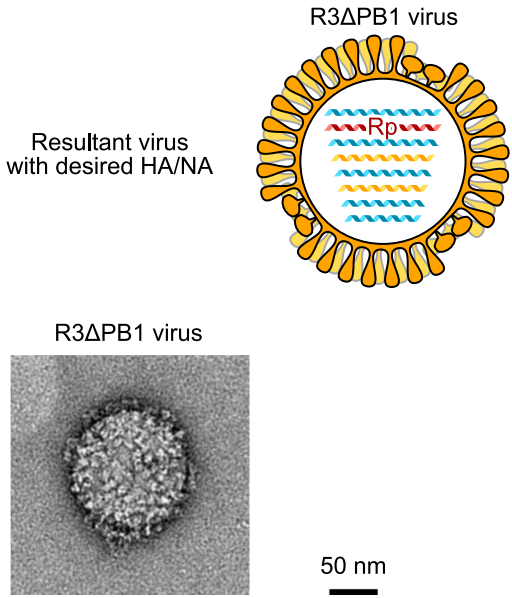

Fig. 1 Development of replication-restricted reporter (R3) $\triangle P B 1$ influenza

A virus. a Design of engineered PB1 segment encoding fluorescence reporter tdKatushka2. b, c Rescue of parental wild-type molecular clone (b) and R3 $\triangle P B 1$ (c) viruses using reverse genetics of eight plasmid system with $\mathrm{HA}$ and NA segments of virus of interest and internal gene segments of $A$ / WSN/1933. PB1 segment of A/WSN/1933 is used to rescue molecularly cloned parental viruses, while engineered PB1 segment is used for R3 $\triangle \mathrm{PB} 1$ influenza viruses. R3 $\triangle P B 1$ influenza viruses require cells expressing PB1 in trans for rescue and propagation. Rp, tdKatushka2 reporter gene-encoded segment. d Negative stain electron microscopy of parental molecular clone (left) and R3 $\mathrm{PB} 1$ (right) of A/Michigan/45/2015 (H1N1) virus. Electron microscopic experiment was performed once and representative image of each virus is shown.

which possess $\mathrm{HA}$ and NA of wild-type viruses and the internal genes including PB1 of A/WSN/1933, and propagated in MDCKSIAT1 cells (Fig. 1b, c).

To examine the morphology of $\mathrm{R} 3 \Delta \mathrm{PB} 1$ virus (A/Michigan/45/ 2015, H1N1), we performed negative stain electron microscopy and found that there was no visible difference in size and spike density between $\mathrm{R} 3 \triangle \mathrm{PB} 1$ and the corresponding wild-type molecularly cloned parental viruses, indicating that the R3 $\triangle \mathrm{PB} 1$ virus remains morphologically indistinguishable from its parental virus (Fig. 1d). Virus growth kinetics of the R3 $\triangle \mathrm{PB} 1$ (A/Michigan/ 45/2015) and its parental viruses in MDCK SIAT1 and PB1expressing MDCK-SIAT1 cells confirmed that the R3 $\triangle \mathrm{PB} 1$ virus replicates only in $\mathrm{PB1}$-expressing cells, whereas parental virus replicates similarly in MDCK-SIAT1 cells with or without PB1 expression (Supplementary Fig. 2). These results demonstrate that the $\mathrm{R} 3 \triangle \mathrm{PB} 1$ viruses are replication-incompetent in cells lacking PB1 expression but they replicate comparably in PB1-expressing cells to corresponding parental viruses carrying PB1 with identical $\mathrm{HA}$ and NA.

Influenza virus neutralization assay using $\mathbf{R} 3$ virus. We tested whether the R3 $\triangle \mathrm{PB} 1$ viruses can be used in an influenza virus neutralization assay. To do so, neutralization assays were carried out utilizing both $\mathrm{R} 3 \triangle \mathrm{PB} 1$ and parental viruses with matched HA and NA, where we compared the inhibitory concentration of a set of mAbs. To verify the reporter-based readout, the $\mathrm{R} 3 \triangle \mathrm{PB} 1$ virusinfected cells were detected either by ELISA with anti-NP antibody or by fluorescence using an automated image-based plate reader at $24 \mathrm{~h}$ post infection. We measured neutralizing activity ( $80 \%$ inhibitory concentration, $\mathrm{IC}_{80}$ ) detected by both ELISAand fluorescent reporter-based readouts for several $\mathrm{mAbs}$ against a total of $8 \mathrm{R} 3 \triangle \mathrm{PB} 1$ viruses (4 H1N1 and $4 \mathrm{H} 3 \mathrm{~N} 2$ viruses) and found a strong positive correlation (Pearson $r=0.94, p<0.001$ ) between the two $\mathrm{IC}_{80}$ datasets, demonstrating that the fluorescent reporter-based readout yields comparable neutralization results to conventional ELISA-based detection method (Fig. 2a and Supplementary Fig. 3).

To assess the $\mathrm{R} 3 \triangle \mathrm{PB} 1$ viruses with the $\mathrm{MN}$ assay format, we next determined neutralizing $\mathrm{IC}_{80}$ for $6 \mathrm{mAbs}$ against matched pairs of $\mathrm{R} 3 \Delta \mathrm{PB} 1$ and parental viruses $(4 \mathrm{H} 1 \mathrm{~N} 1$ and $3 \mathrm{H} 3 \mathrm{~N} 2$ viruses). Cells infected with $\mathrm{R} 3 \Delta \mathrm{PB} 1$ viruses were detected by fluorescence, while cells infected with parental viruses were detected by ELISA. The positive correlation (Pearson $r=0.87, p$ $<0.001$ ) between neutralization $\mathrm{IC}_{80}$ of $\mathrm{mAbs}$ against $\mathrm{R} 3 \Delta \mathrm{PB} 1$ and parental viruses shows that $\mathrm{R} 3 \Delta \mathrm{PB} 1$ viruses retained the neutralization sensitivity of parental viruses with matched HA and NA (Fig. 2b). In conclusion, our results indicate that the neutralization assay using $\mathrm{R} 3 \triangle \mathrm{PB} 1$ viruses with fluorescencebased readout can be used as high-throughput, safe, and reliable measurement of virus-neutralizing activity.

Building a comprehensive panel of $\mathbf{R} 3$ viruses. We aimed to build a comprehensive panel of $\mathrm{R} 3 \Delta \mathrm{PB} 1$ viruses spanning the entire antigenic evolution of human $\mathrm{H} 1 \mathrm{~N} 1$ and $\mathrm{H} 3 \mathrm{~N} 2$ subtype viruses. We selected representative influenza strains based on phylogenetic analysis of HA sequences deposited in public databases, literatures on genetic and antigenic evolution of human $\mathrm{H} 1 \mathrm{~N} 1$ and $\mathrm{H} 3 \mathrm{~N} 2$ influenza viruses, and vaccine strains utilized since $1930 \mathrm{~s}^{23,25-28}$.

H1N1 subtype virus was introduced into the human population in 1918 and circulated until it was replaced by H2N2 virus in 1957. H1N1 virus re-emerged in 1977 and circulated until 2009. During this period, H1N1 viruses evolved significantly through genetic drift into multiple clades with distinct genetic and antigenic properties ${ }^{27,29-31}$. To capture the antigenic variations of these viruses, we chose 7 matched HA and NA sequences from viruses circulating between 1933 and 1957 and $13 \mathrm{HA}$ and NA sequences from viruses circulating between 1977 and 2009. The 2009 pandemic H1N1 virus acquired sustained human-to-human transmission and rapidly and completely replaced the prepandemic H1N1 virus. Since its emergence, the 2009 pandemic H1N1 virus has accumulated several amino acid substitutions linked to changes in antigenicity 32 . Therefore, we included $3 \mathrm{HA}$ and NA sequences from viruses isolated between 2009 and 2015 and $\mathrm{HA}$ and NA sequences of swine-origin A/New Jersey/8/1976 (H1N1), which caused an isolated outbreak in 1976 in the United States. In summary, our H1N1 panel consists of 19 pre-pandemic strains, 3 pandemic strains, and 1 swine-origin H1N1 strain (Fig. 3a). Of note, our panel of $\mathrm{H} 1 \mathrm{~N} 1$ viruses includes genetically similar viruses to all World Health Organization (WHO)-recommended H1N1 vaccine 
$\mathbf{a}$

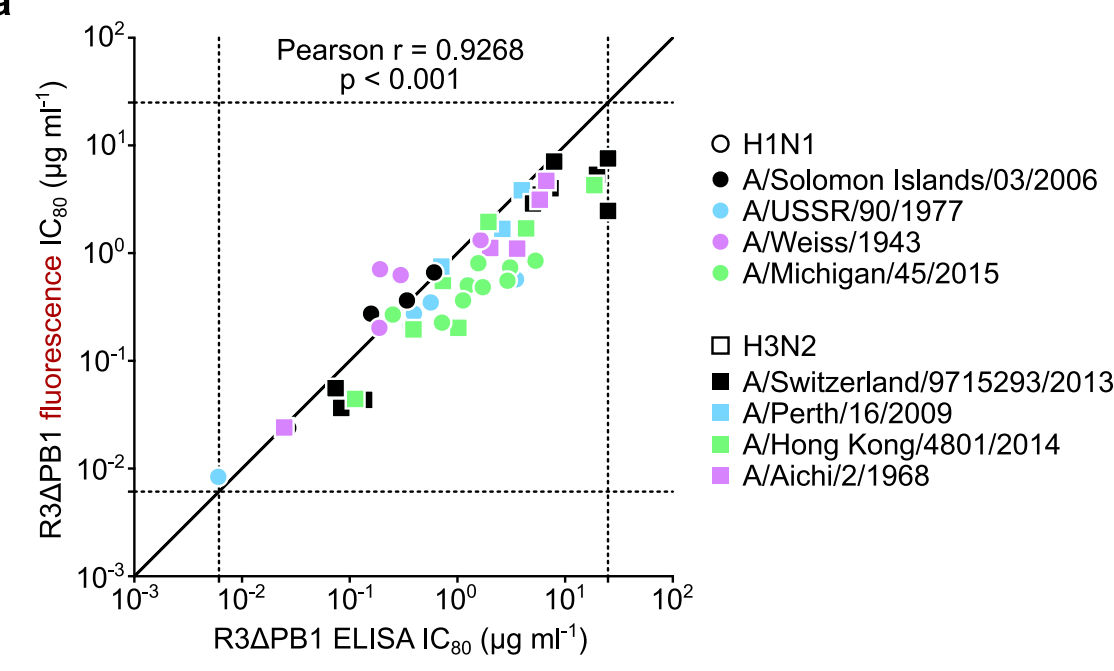

b

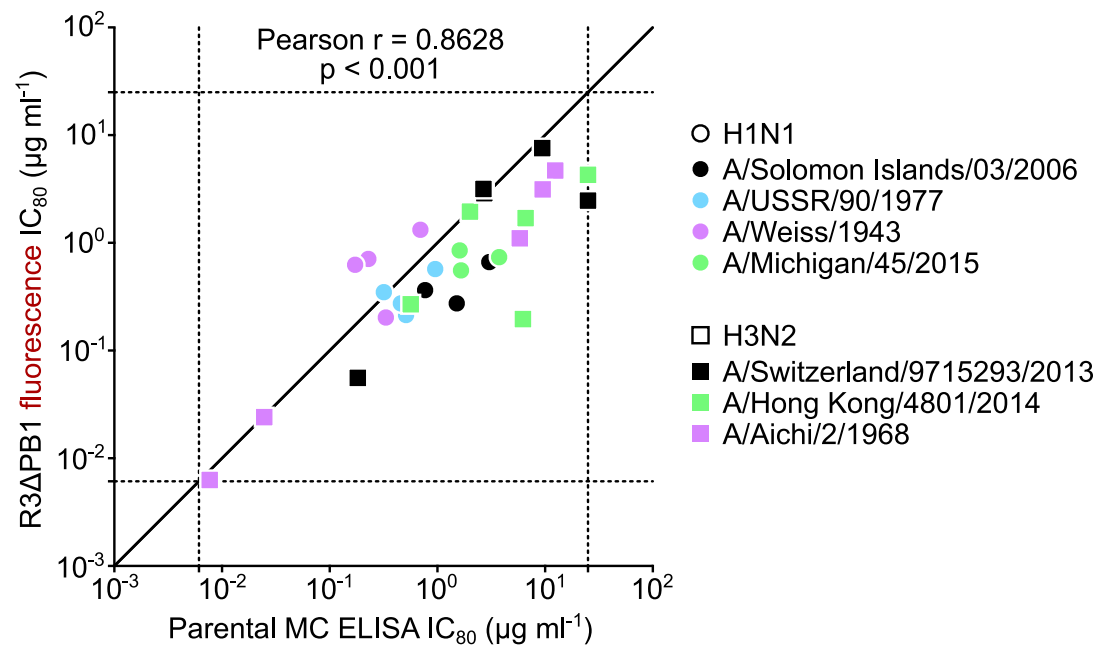

Fig. 2 Assessment of R3 $\triangle$ PB1 virus for neutralization assay. a Correlation between fluorescence- and ELISA-based readouts. Each dot indicates the neutralization titers $\left(\mathrm{IC}_{80} \mu \mathrm{g} \mathrm{m \textrm {m } ^ { - 1 }}\right.$ ) of a single monoclonal antibody against an R3 $\Delta \mathrm{PB} 1$ influenza virus measured by fluorescence readout ( $y$-axis) and ELISA with anti-NP antibody ( $x$-axis). b Correlation between assays using R3 $\triangle \mathrm{PB} 1$ and molecular clone (MC) viruses. Each symbol indicates the

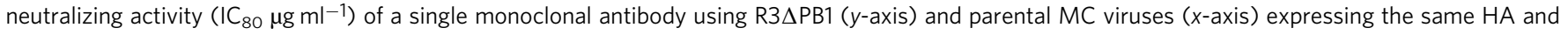
NA. Colors indicate different viruses. Circles and squares represent H1N1 and H3N2 viruses, respectively. Dashed lines indicate upper and lower limits of detection. Pearson $r$ correlation coefficient and two-tailed $p$ values for each analysis are shown above each graph. Neutralization assays were performed at least twice and representative data are plotted.

strains between 1977 and 2015 (fludb.org/brc/vaccineRecommend. spg?decorator=influenza), antigenically representative viruses circulating in humans in the $1940 \mathrm{~s}^{31}$, or representatives of the major HA and NA lineages of seasonal $\mathrm{H} 1 \mathrm{~N} 1$ viruses $^{30}$.

H3N2 subtype virus has been circulating in humans since its emergence in 1968. Comprehensive genetic and antigenic analysis of human $\mathrm{H} 3 \mathrm{~N} 2$ viruses groups them into 14 distinct antigenic clusters $^{21,26,28,33}$, and hence, we include several representatives from each antigenic cluster in our panel. Sporadic outbreaks with swine $\mathrm{H} 3 \mathrm{~N} 2$ variant $(\mathrm{H} 3 \mathrm{~N} 2 \mathrm{v})$ have also been described in humans ${ }^{34}$. Therefore, we included the swine-origin $\mathrm{H} 3 \mathrm{~N} 2 \mathrm{v}$ strain, A/Indiana/10/2011. As a result, our H3N2 panel includes 25 human $\mathrm{H} 3 \mathrm{~N} 2$ strains and $1 \mathrm{H} 3 \mathrm{~N} 2 \mathrm{v}$ strain (Fig. 3b).

When we calculate the conservation of solvent-exposed surface among $\mathrm{H} 1$ and $\mathrm{H} 3 \mathrm{HAs}$ included in our panel, we notice that the head region of $\mathrm{HA}$ of both $\mathrm{H} 1$ and $\mathrm{H} 3$ is substantially more variable while the stem region is mostly conserved as expected (Fig. 3c, d). The degree of surface conservation reflects that of much larger datasets of $\mathrm{H} 1$ and $\mathrm{H} 3 \mathrm{HAs}$ (Fig. 3c, d), suggesting that the selected HA sequences in our R3 virus panel capture the $\mathrm{HA}$ diversity of human $\mathrm{H} 1 \mathrm{~N} 1$ and $\mathrm{H} 3 \mathrm{~N} 2$ viruses.

Generation of R3 $\Delta \mathrm{HA}$ viruses with highly pathogenic influenza virus sequences. Working with highly pathogenic influenza viruses (e.g., H5N1, H7N9, $1918 \mathrm{H} 1 \mathrm{~N} 1$ ) or influenza lineages disappeared from human population (e.g., H2N2) requires high containment laboratories. Although $\mathrm{R} 3 \Delta \mathrm{PB} 1$ viruses have limited capacity to replicate due to the requirement of PB1 complementation, they retain the ability to reassort the HA segment with wild-type influenza viruses. To prevent this event, we evaluated alternative approaches less susceptible to reassortment and generated reporter viruses expressing HA and NA of viruses with pandemic potential ${ }^{19,35}$.

First, we developed influenza viruses, unable to reassort HA segments, by making "rewired" R3 $\triangle \mathrm{PB} 1$ viruses (R4 $\Delta \mathrm{PB} 1)$ as previously described ${ }^{35}$. For this purpose, two segments of influenza genome were altered: the PB1 segment was modified to encode HA, 

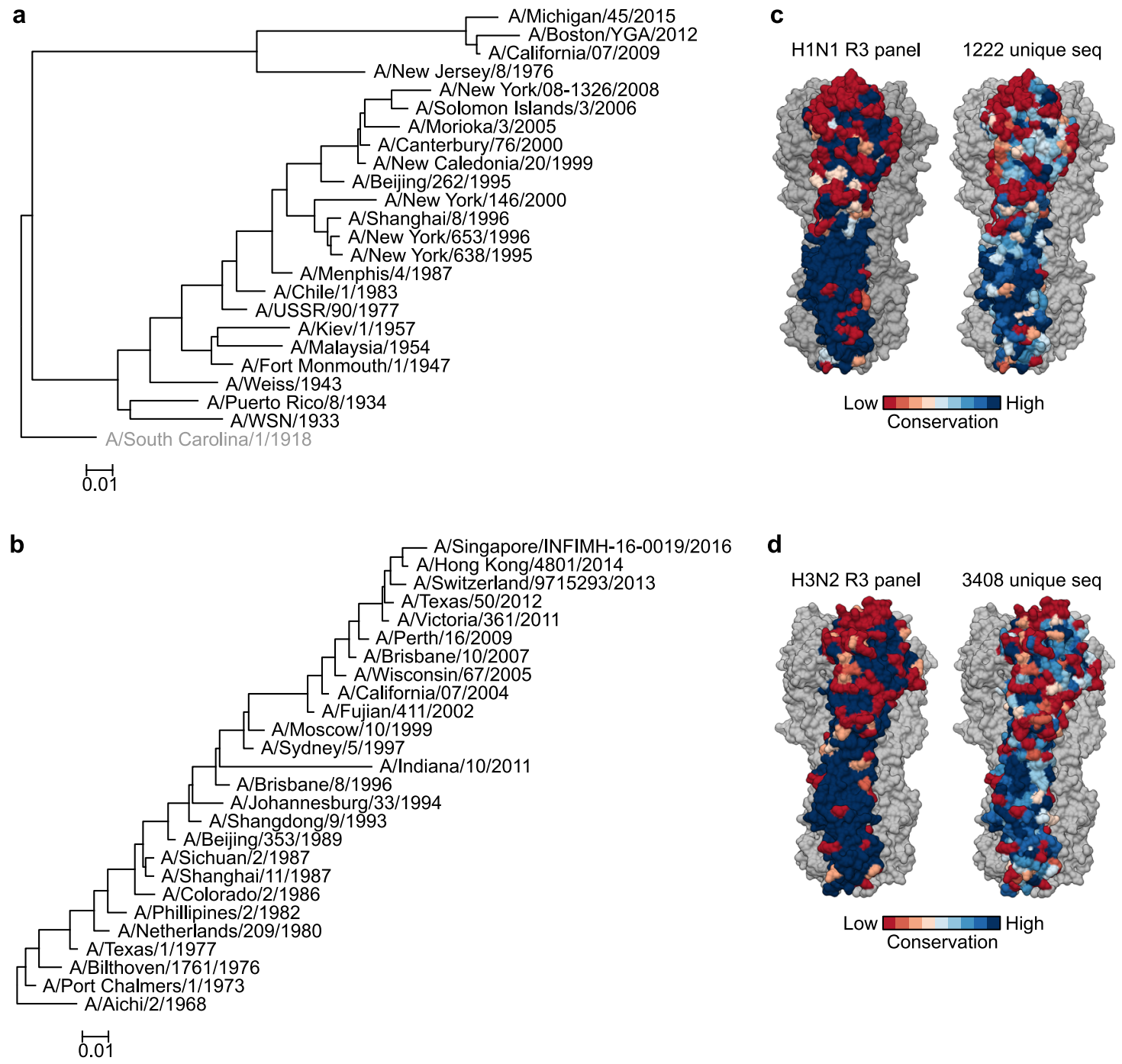

$$
\text { A/Port Chalmers/1/1973 }
$$$$
5.01
$$

Fig. 3 Phylogenetic and protein surface conservation analysis of HA of H1N1 and H3N2 viruses used in the study. a, b Phylogenetic tree of 24 H1 (a) and $26 \mathrm{H3}$ (b) HA sequences used in the R3 panel. The tree was generated by NJ method and rooted with A/South Carolina/1/1918 (a) (not used in the study) or with $\mathrm{A} /$ Aichi/2/1968 (b). Distance scale bar indicates $1 \%$ difference in nucleotide identity. c, d Protein surface conservation of $\mathrm{H} 1$ (c) and $\mathrm{H} 3$ (d) HA used in the R3 panel and a larger non-redundant representative dataset. Amino acid conservation at each residue was calculated and scored by using the Consurf server (https://consurf.tau.ac.il/) and rendered on the structure of A/California/07/2009 (PDB: 3LZG) (c) and A/Victoria/361/2011 (PDB:4WE8) (d). Conservation at each position was scored 1-9 (lowest to highest) and colored according to the conservation score on one protomer of HA trimer. Two other HA protomers are colored in gray.

and the HA segment was modified to encode the tdKatushka2 reporter. Using these altered PB1 and HA segments and reverse genetics, we were able to rescue R4 $\triangle \mathrm{PB} 1 \mathrm{~A} / \mathrm{Switzerland} / 9715293 /$ 2013 (H3N2) virus in PB1-expressing cells (Supplementary Fig. 4a \pm c). By measuring neutralizing $\mathrm{IC}_{80}$ of $15 \mathrm{mAbs}$ for both $\mathrm{R} 3 \Delta \mathrm{PB} 1$ and $\mathrm{R} 4 \Delta \mathrm{PB} 1$, we found a strong positive correlation (Pearson $r=0.94$, $p<0.001$ ) between $\mathrm{IC}_{80}$ neutralizing activity against the two viruses, demonstrating that these viruses have equivalent neutralization sensitivities (Supplementary Fig. 4d). Moreover, we found that the $\mathrm{R} 4 \Delta \mathrm{PB} 1 \mathrm{~A} /$ Switzerland/9715293/2013 (H3N2) virus did not reassort its HA segment with A/Solomon Islands/03/2006 (H1N1) when the two viruses were co-infected and propagated on PB1-expressing cells (Supplementary Fig. 4e). Although these results show that R4 $\triangle \mathrm{PB} 1$ viruses can be safely rescued and used for high-throughput neutralization assay, further work is required to optimize the rescue and the propagation of $\mathrm{R} 4 \triangle \mathrm{PB} 1$ to be a practical and deployable process for producing reporter viruses.

Next, we explored an alternative approach to rescue R3 viruses unable to reassort the HA segment by replacing the
HA coding sequence with the reporter gene (R3 $\Delta H A)$. In this configuration, the virus lacks a functional HA segment and is unable to reassort the segment with other viruses (Fig. $4 \mathrm{a}-\mathrm{c}$ ), and it will be able to replicate only in HAexpressing cells ${ }^{19}$. To assess $\mathrm{R} 3 \Delta \mathrm{HA}$ viruses for the reporter neutralization assay, we generated R3 $\Delta \mathrm{HA}$ A/Switzerland/ $9715293 / 2013$ (H3N2) virus to compare the neutralization $\mathrm{IC}_{80}$ of several mAbs against both $\mathrm{R} 3 \Delta \mathrm{PB} 1$ and $\mathrm{R} 3 \Delta \mathrm{HA}$ viruses. There was a positive correlation between neutralization $\mathrm{IC}_{80}$ determined with these viruses (Pearson $r=0.90, p<0.001$ ), although we noticed slight differences in sensitivity to neutralization when testing anti-stem antibodies against $\mathrm{R} 3 \Delta \mathrm{PB} 1$ and $\mathrm{R} 3 \Delta \mathrm{HA}$ viruses (Fig. 4d). Using this approach, we prepared $6 \mathrm{R} 3 \Delta \mathrm{HA}$ viruses (i.e., $2 \mathrm{H} 5 \mathrm{~N} 1$ of clade $1, \mathrm{~A} /$ Vietnam/1203/2004, and clade 2.1.3, A/Indonesia/05/2005, 2 genetically and antigenically related H7N9, A/Anhui/01/2013 and A/Shanghai/02/2013, 1 H2N2, A/Singapore/1/1957, and 1 H10N8, A/Jiangxi-Donghu/346-2/2013) in addition to the 49 $\mathrm{R} 3 \Delta \mathrm{PB} 1$ virus panel ${ }^{36,37}$. 
a

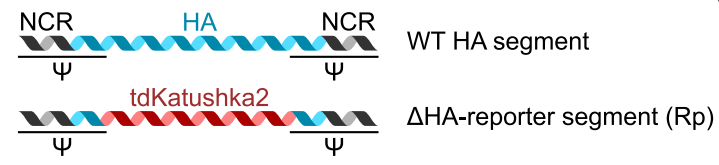

b

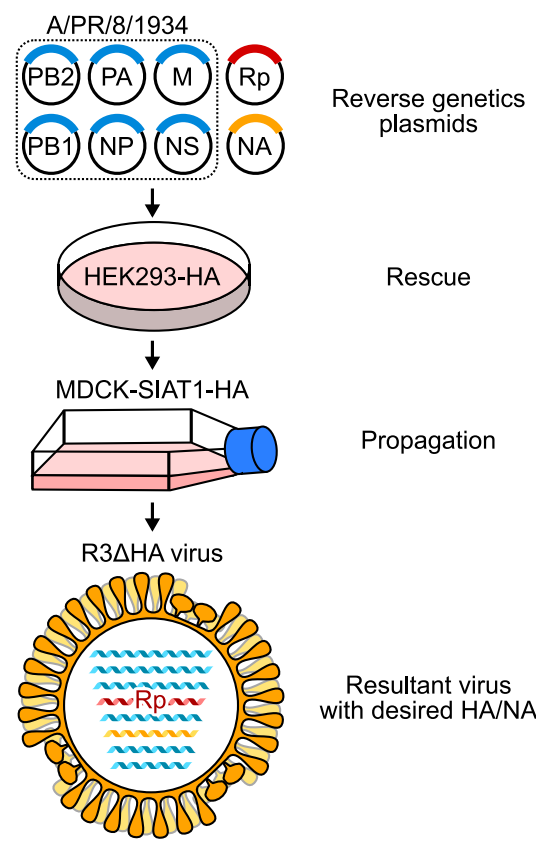

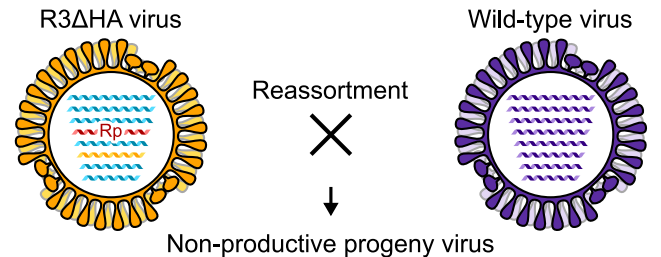

Non-productive progeny virus

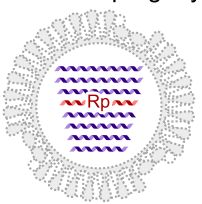

d

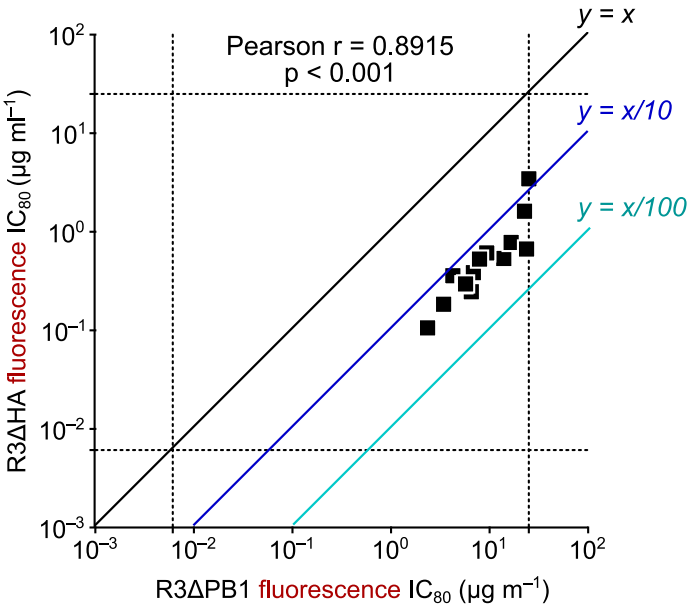

Fig. 4 Development of R3 $\Delta$ HA influenza viruses. a Design of $H A$ segment used to rescue R3 $\Delta H A$ virus. $\mathbf{b} 3 \Delta H A$ virus requires cells expressing $H A$ protein in trans for rescue and propagation. c Non-viable reassortment between R3 $\Delta \mathrm{HA}$ and wild-type influenza viruses. Reassortant virus carrying the engineered HA segment encoding the reporter gene results in replication-deficient virus. Rp, tdKatushka2 reporter gene-encoded segment. $\mathbf{d}$ Neutralization sensitivity of R3 $\Delta \mathrm{HA}$ viruses. Correlation between neutralization titers of 15 monoclonal antibodies against R3 $\Delta \mathrm{PB} 1$ and R3 $\mathrm{HA}$ of A/Switzerland/9715293/

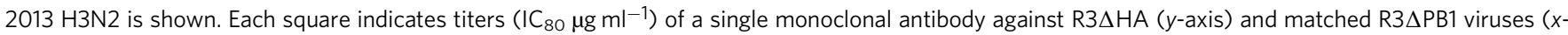
axis). Dashed lines indicate upper and lower limits of detection. Neutralization assays were performed at least twice and representative data are plotted.

Characterizing influenza responses in serum samples from human vaccinees using $\mathbf{R} 3$ viruses. To assess neutralization activity in polyclonal sera using R3 viruses, we measured the neutralization titers in samples collected in the Phase I human clinical trials of experimental $\mathrm{H} 5 \mathrm{~N} 1$ and $\mathrm{H} 7 \mathrm{~N} 9$ vaccines conducted by the Vaccine Research Center in 2011 and 2015, respectively (NCT01086657 and NCT02206464, respectively). Briefly, healthy adult volunteers were immunized with DNA encoding H5 (A/ Indonesia/05/2005) or H5N1 monovalent influenza vaccine (MIV), or DNA encoding H7 (A/Shanghai/02/2013) HA, H7N9 MIV, or combinations of H7 DNA and H7N9 MIV followed by a boost with H5N1 MIV or H7N9 MIV, respectively ${ }^{38,39}$. Neutralization titers were measured at the time of vaccination (week 0 ) and 2 weeks post-boost against vaccine-matched strain (i.e., H5N1 A/Indonesia/ 05/2005 or H7N9 A/Shanghai/02/2013 R3 $\Delta \mathrm{HA}$ ), two H1N1 R3 $\triangle \mathrm{PB} 1$ (A/Michigan/45/2015 and A/New Caledonia/20/1999), and two H3N2 R3 $\triangle \mathrm{PB} 1$ (A/Hong Kong/4801/2014 and A/Shangdong/9/1983) viruses. For individuals vaccinated with the $\mathrm{H} 5$ prime-boost regimen ${ }^{38}$, we found that neutralization titers against the vaccine strain $\mathrm{H} 5 \mathrm{~N} 1 \mathrm{~A} /$ Indonesia/05/2005 increased at 2 weeks post-boost from a baseline reciprocal $\mathrm{IC}_{80}$ GMT of 727.8 [range 206.6-1603.5] to a final reciprocal $\mathrm{IC}_{80}$ GMT of 33,014.2 [range $3235.7-223,905.4] \quad(p<0.0001)$. We also observed a significant increase $(p=0.0003)$ in the reciprocal $\mathrm{IC}_{80}$ titers against H1N1 A/ Michigan/45/2015 virus (Supplementary Fig. 5a). Similarly, for individuals vaccinated with the $\mathrm{H} 7$ prime-boost regimen ${ }^{39}$, we found that titers against H7N9 A/Shanghai/02/2013 virus had increased significantly upon vaccination (from a baseline reciprocal
$\mathrm{IC}_{80}$ GMT of 246.9 [range <40-1192.1] to 3134 [range 765.2-31,285.8] ( $p=0.0013)$. Of note, the H7 prime-boost regimen did not increase significantly neutralization titers against two H1N1 nor two H3N2 viruses tested in any of these individuals (Supplementary Fig. 5b). Together, these results establish that the neutralization assay using R3 viruses can be used to measure the influenza virus-neutralizing activities in human polyclonal sera.

Measurement of the viral inhibitory activity of anti-NA antibodies using R3 influenza virus. To assess the viral inhibitory activity of anti-NA antibodies, we adapted our assay using the R3 viruses by adding a thixotropic overlay and quantifying the virus-infected area (i.e., fluorescent plaques). Broadly crossprotective anti-NA $\mathrm{mAb} 1 \mathrm{G} 01^{40}$ and murine anti-NA mAb $\mathrm{CD}^{41}$ inhibited virus propagation in a concentration-dependent manner while non-targeting mAb D25 (anti-respiratory syncytial virus $)^{42}$ had no effect on propagation of R3 $\triangle \mathrm{PB} 1 \mathrm{~A} / \mathrm{California/}$ 07/2009 H1N1 viruses (Supplementary Fig. 6). These results suggest that our fluorescent plaque-reduction assay with influenza R3 viruses allows live, high-throughput measurement of viral inhibitory activities targeting NA and will facilitate the characterization of novel vaccine candidates and $\mathrm{mAbs}$ with antiNA responses.

Profiling of influenza neutralizing $\mathrm{mAbs}$ with a 55-virus panel. Using our comprehensive panel of R3 influenza viruses spanning 6 different influenza A subtypes, we profiled the neutralization 


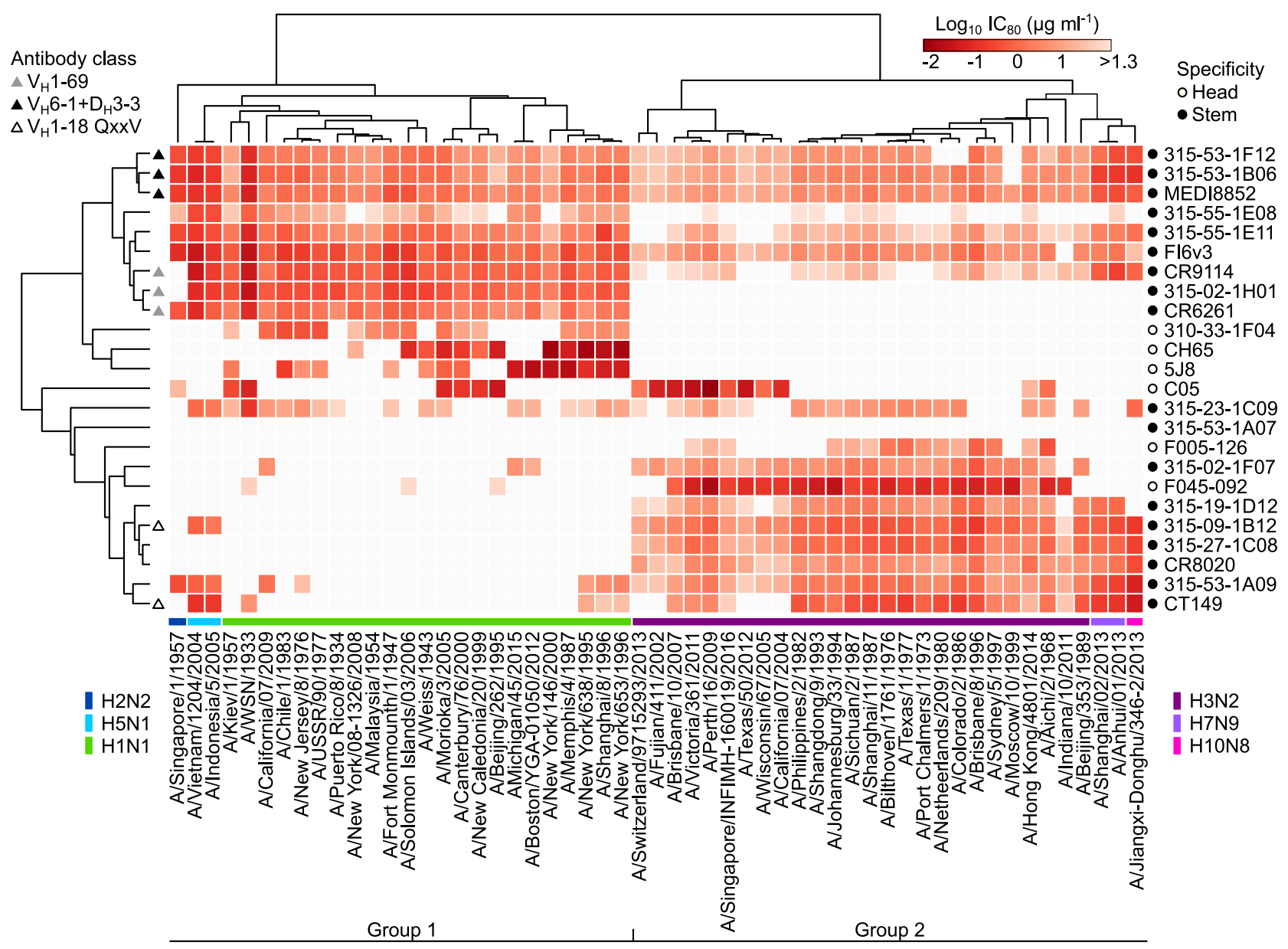

Fig. 5 In-depth neutralization profiling of influenza monoclonal antibodies. Heatmap of neutralization titers $\left(\mathrm{IC}_{80} \mu \mathrm{g} \mathrm{ml}^{-1}\right)$ of 24 monoclonal antibodies against a panel of 55 R3 influenza A viruses. Virus subtypes are color-coded and indicated the bottom of the heatmap. Open and closed circles indicate specificity of monoclonal antibodies and are shown on the right of the heatmap (open, HA head-; closed, HA stem-directed). Convergent classes of bnAbs with specific gene signatures are indicated on the left of the heatmap as triangles (gray, $V_{H} 1-69$; black, $V_{H} 6-1+D_{H} 3-3$; open, $V_{H} 1-18$ QxxV). Heatmap and unsupervised clustering was made with the ClustVis server (https://biit.cs.ut.ee/clustvis/). Note: H1N1 and H3N2 viruses are R3 $\Delta$ PB1 and all other viral subtypes are R3 $\triangle \mathrm{HA}$ viruses. Neutralization assays were performed at least twice and representative data are plotted.

breadth and potency of a total of 24 human mAbs. Thirteen of them were isolated at the Vaccine Research Center from peripheral blood mononuclear cells collected as part of the H5N1 or H7N9 vaccine clinical trials $39,43-45$, while 11 other antibodies were previously described elsewhere ${ }^{46-55}$. Among 24 antibodies, 18 antibodies recognize epitopes on the conserved HA stem region and 6 antibodies (i.e., CH65, 5J8, C05, F045-092, F005126 , and 310-33-1F04) bind within the HA head region. HA stem-binding 315-53-1A07 which did not neutralize any of the viruses was included in our panel. Of note, neutralization $\mathrm{IC}_{80}$ values of the mAbs did not change drastically between 18 and 24 $\mathrm{h}$ after infection while the virus-infected cells dramatically increased during this time period (Supplementary Fig. 7), providing a reasonable flexibility for assay operation and fluorescent readout. We generated a matrix of neutralizing profiles for 24 antibodies against $55 \mathrm{R} 3$ viruses consisting of 1320 data points and analyzed the matrix by hierarchical clustering (Fig. 5). Despite the fact that there is no associated information about viruses in the input dataset, the profile matrix segregates not only groups of viruses but also virus subtypes into defined clusters (Fig. 5). Interestingly, the hierarchical clustering groups several antibodies according to their immunogenetic composition or convergent antibody class ${ }^{44,56,57}$. For example, MEDI8852 is clustered with 2 other antibodies (315-53-1F12 and 315-53-1B06) and all the three antibodies belong to the $\mathrm{V}_{\mathrm{H}} 6-1+\mathrm{D}_{\mathrm{H}} 3-3$ convergent multi-donor class $^{57}$, while CR9114 is clustered with CR6261 and 315-02-1H01, which all shares $V_{H} 1-69$. The latter case is particularly noteworthy as CR9114 has much broader neutralizing capacity than the other two, yet clustered with the stereotypical group 1-specific $\mathrm{V}_{\mathrm{H}} 1-69$ antibodies CR6261 and 315-02-1H01 (Fig. 5).

Deep characterization of neutralizing profiles also predicts the developmental pathway for each bnAb. The convergent multidonor bnAbs in $\mathrm{V}_{\mathrm{H}} 1-18$ QXXV class (i.e., 315-09-1B12 and CT149) neutralize many group 2 viruses while having very limited breadth against group 1 viruses (Fig. 5). This confirms previous findings in which the unmutated common ancestor (UCA) of this class of bnAbs engages only group 2 HAs and acquires group 1 reactivity through somatic hypermutation ${ }^{57}$. Conversely, the $\mathrm{V}_{\mathrm{H}} 6-1+\mathrm{D}_{\mathrm{H}}$ 3-3 class bnAbs (i.e., MEDI8852, 315-53-1F12, and 315-53-1B06) possess higher neutralization potency against group 1 viruses than group 2 viruses (Fig. 5), and this is consistent with the preferential engagement of group 1 HAs to the UCAs of this class ${ }^{57}$. This deep neutralization profiling dataset also allows us to generate neutralization breadth-potency curves at relatively high resolution (Fig. 6). Previous studies used a dataset generated by pseudotyped lentiviral neutralization assays with 15-17 selected HA-NA 


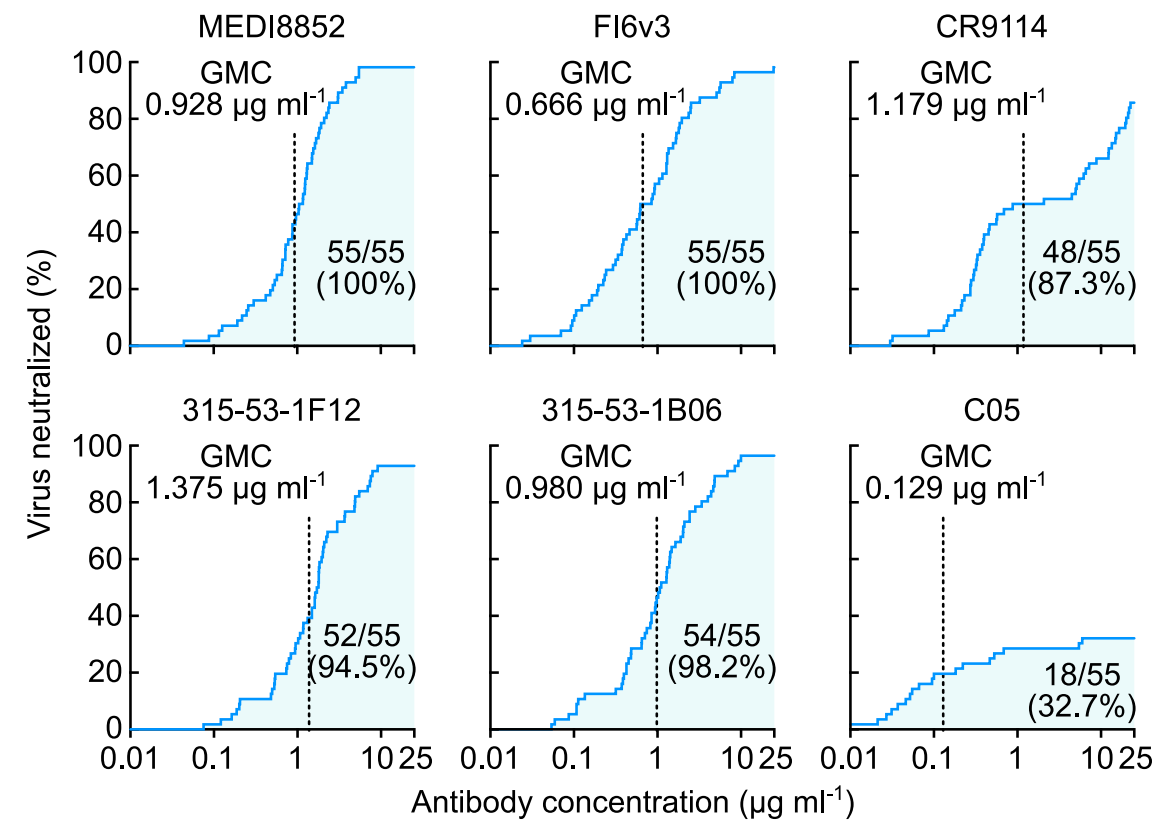

Fig. 6 Neutralization breadth-potency analysis of broadly cross-reactive stem-directed antibodies and highly potent receptor-binding site antibody. Neutralization titers $\left(\mathrm{IC}_{80} \mathrm{\mu g} \mathrm{ml}^{-1}\right.$ ) against a panel of $55 \mathrm{R} 3$ viruses were used to generate the breadth-potency plot for each antibody. Geometric mean $\mathrm{IC}_{80}$ concentration (GMC) of each antibody was calculated only for the viruses neutralized by given antibody and indicated as a vertical dashed line on each graph. Shaded areas correspond to the number of viruses neutralized by given antibody. Both the number of viruses neutralized out of 55 R3 viruses and neutralization coverage (\%) are indicated on each graph.

sequences ${ }^{45,57}$ and provided a limited understanding of neutralization breadth both because of the relatively small number of strains and the hypersensitivity of the pseudotyped lentivirus assay format. By determining the neutralization profiles using our comprehensive R3 virus panel, we found two antibodies (MEDI8852 and FI6v3) that were capable of neutralizing all 55 viruses (Fig. 6). The other two $\mathrm{V}_{\mathrm{H}} 6-1+\mathrm{D}_{\mathrm{H}} 3-3$ class mAbs, 31553-1F12 and 315-53-1B06, neutralized 52 (94.5\%) and 54 (98.2\%) of 55 viruses, respectively. Although CR9114 neutralized 48 out of 55 viruses $(87.3 \%)$ its breadth-potency plot demonstrated a biphasic curve, highlighting the preferential neutralization of group 1 viruses (the first phase) and lower potency against group 2 viruses (the second phase) by this antibody (Fig. 6). The RBSdirected antibody $\mathrm{C} 05$ showed limited neutralization breadth (32.7\%) yet was extremely potent (Fig. 6).

Overall, the neutralization profiling performed with the reporter influenza virus assay provides less biased breadth and potency information than the pseudotyped lentivirus assay or highly variable traditional MN assay. High-resolution information may also help predict the class and the origin of antibodies with particular neutralization signatures.

\section{Discussion}

Current influenza vaccines are imperfect and there is a large room to improve their efficacy, consistency, and breadth. Historically, efficacy has been associated with serum HAI activity ${ }^{58,59}$ and the HAI assay was developed as a surrogate for virus neutralization ${ }^{60,61}$. To improve current vaccines and to develop new vaccine concepts that will have broader and more durable efficacy, accurate measurement of neutralizing activity across a broad range of influenza viruses is critical. The HAI assay is a surrogate only for neutralization directed at the RBS on HA, therefore neutralization targeting the HA stem or other relatively conserved sites on HA and NA will not correlate with HAI. In addition, some new drifted seasonal strains of influenza and subtypes with pandemic potential do not always have predictable hemagglutination properties. Therefore, to encompass the variety of $\mathrm{HA}$ and NA strains and the antibodies induced by diverse vaccine approaches, emphasis on a reproducible and authentic measurement of virus-neutralizing activity is needed.

Unlike the HAI assay that measures the antibody's ability to block receptor binding of viral $\mathrm{HA}$, plaque reduction ${ }^{62}$ and $\mathrm{MN}^{63}$ assays can capture neutralizing antibodies that block any virus replication step, including attachment, internalization, $\mathrm{pH}$ induced conformational change of $\mathrm{HA}$, membrane fusion, and virus egress. Despite efforts initiated by WHO, execution of influenza neutralization assays is still not standardized, leading to significant variability among results reported by different laboratories ${ }^{9,63}$. Development of a large panel of representative influenza viruses and significant improvement of assay throughput and safety are highly anticipated in the field and will enable deep characterization and systematic comparisons of influenza virus-neutralizing antibody responses. The virus panel combined with serum and mAb standards will allow normalization across assay platforms and facilitate the evaluation of universal influenza vaccine candidates. In the present study, we address these outstanding needs by building an engineered influenza reporter virus-based neutralization assay coupled with high-throughput image-based readout in a biosafety level 2 setting. Our panel consists of 55 reporter viruses capturing almost the entire antigenic evolution of human $\mathrm{H} 1 \mathrm{~N} 1$ and $\mathrm{H} 3 \mathrm{~N} 2$ subtypes as well as historical human $\mathrm{H} 2 \mathrm{~N} 2$ and three other subtypes circulating at the human-animal interface. Moreover, we were able to rescue and propagate $\mathrm{R} 3$ chimeric influenza viruses encoding the HA and NA of influenza B using the internal genes of A/WSN/1933 influenza A virus. $\mathrm{R} 3 \triangle \mathrm{PB} 1$ chimeric influenza $\mathrm{A} / \mathrm{B}$ viruses can replicate only in cells expressing influenza A PB1 in trans. This approach to rescue viruses expressing $\mathrm{HA}$ and NA of influenza $\mathrm{B}$ viruses was described in several previous reports ${ }^{64-66}$, and it was shown that chimeric influenza A/B viruses have similar growth profiles as wild-type influenza $B$ viruses ${ }^{66}$. Although none of the 24 antibodies included in our study neutralized these $\mathrm{R} 3 \Delta \mathrm{PB} 1$ chimeric influenza A/B viruses, we have successfully utilized these 
viruses in neutralization assays to assess anti-influenza B virusneutralizing antibody responses elicited by experimental vaccines $^{67}$.

Direct detection of fluorescent reporter allowed us to measure virus replication in live cells without additional signal amplifications unlike ELISA- or hemagglutination-based readout. Imagebased readout was chosen to further facilitate fast and precise data acquisition and analysis. In contrast to ELISA-based assays, in which the signal is measured for the entire well after a series of signal amplification steps, image-based detection provides higher signal-to-noise ratio, dynamic range, and precision at single-cell resolution. The R3 virus neutralization assays can be readily automated to minimize hands-on time and human errors. It is also worth noting that the fluorescent image-based readout makes the assay more cost-effective compared with other assays such as ELISA- and luciferase-based assays. By using R3 viruses, we can detect virus-inhibitory activity of both anti-HA and anti-NA antibodies by counting number of fluorescent cells or measuring size of fluorescent plaques in a safe and high-throughput manner.

Recent attempted improvements of the WHO-recommended ELISA-based MN assay protocol ${ }^{68-70}$ underscore the need for the development of more reliable assays. As noted, previous reports have described the potential utility of R $3 \Delta P B 1^{18}$ and $R 3 \Delta H A^{19,20}$ influenza viruses in neutralization assays. However, these efforts did not lead to widespread adoption and the development of standardized influenza neutralization assays due to the small number of available reporter viruses, impediments to access essential reagents (e.g., influenza reverse genetics plasmids and stable cell lines), and lack of comprehensive validation. We found that the antigenicity and neutralization sensitivity of R3 $\triangle \mathrm{PB} 1$ is similar to that of authentic influenza viruses, while $\mathrm{R} 3 \Delta \mathrm{HA}$ viruses appeared to be slightly more sensitive to neutralization than $\mathrm{R} 3 \triangle \mathrm{PB} 1$. This is likely due to less efficient HA incorporation into R3 $\Delta \mathrm{HA}$ viruses ${ }^{19,20}$ and represents a common feature of $\mathrm{R} 3 \Delta \mathrm{HA}$ viruses. Although we noticed that the neutralization sensitivity of $\mathrm{R} 4 \Delta \mathrm{PB} 1$ closely matched that of $\mathrm{R} 3 \Delta \mathrm{PB} 1$ implying their potential superiority for building viruses that are unlikely to reassort, additional optimization of $\mathrm{R} 4 \Delta \mathrm{PB} 1$ virus is necessary to become a practical alternative due to its poor rescue efficiency. Although the use of R3 (and R4) viruses offers further advancements in the assay throughput, safety, and precision, this approach has its own limitations. Since the reporter segment is not required for viral replication, it is inevitable that after extensive virus passage, the reporter segment may accumulate mutations made by the error-prone viral RNA polymerase, which in turn, will diminish reporter gene activity and/or expression. However, it is possible to prepare virus stocks relatively quickly with five or less passages, and we confirm that these stocks retain an active reporter gene expression. Additionally, genomic stability of R3 influenza viruses can be improved by utilizing the variant PB1 polymerase with lower error rates as reported recently ${ }^{71}$ and designing R3 viruses capable of replicating only when the engineered genomic segment is functional, such as inducible geneexpression systems.

As we continue to expand our collection of viruses and neutralizing antibodies, we will be able to perform neutralization fingerprint analysis ${ }^{72}$ to provide a better understanding of the relationships between fine epitope specificity and neutralization breadth and potency and allow computational predictions of the epitope-specific contributions of polyclonal serum antibodies to overall neutralizing activity. This type of analysis will foster the immune monitoring of antibody responses elicited by universal influenza vaccine candidates, particularly those targeting the conserved HA stem supersite $\mathrm{T}^{73-75}$. By describing our methods and depositing sequences required to generate R3 viruses, we provide the basis for implementing this technology for the strains reported here as well as for future emerging viruses. We anticipate that our assay will foster rapid discovery and characterization of influenza bnAbs as well as virus-inhibitory antibodies and facilitate efficient evaluation of vaccine-elicited antibody responses for accelerating the efforts to develop universal influenza vaccines.

\section{Methods}

Plasmids. To prepare influenza reverse genetics plasmids for rescue of influenza A $\mathrm{H} 1 \mathrm{~N} 1$ or H3N2 viruses described in this study, HA and NA coding sequences were retrieved from Genbank; NCRs of A/WSN/1933 for H1N1 or A/Netherlands/009/ 2010 for H3N2 viruses were added at both ends. Full-length HA and NA sequences were cloned into a dual promoter influenza reverse genetics plasmid previously described $^{76}$. To rescue influenza viruses, dual promoter plasmids encoding internal genes of A/WSN/1933 (pHW181-PB2, pHW182-PB1, pHW183-PA, pHW185-NP, pHW187-M, pHW188-NS) were used ${ }^{76}$. To prepare PB1 reporter segment used to rescue $\mathrm{R} 3$ viruses, the sequence containing the $\mathrm{PB} 1$ genome packaging signals of $\mathrm{A}$ WSN $/ 1933^{22}$ and mKate 2 or tdKatushka2 reporter coding region ${ }^{23}$ (Addgene Cat No. 56049) was synthesized and cloned using BsmBI (New England Biolabs) restriction sites into the dual promoter influenza reverse genetics plasmid. Similarly, HA reporter segment was synthesized with HA genome packaging signals of A/Puerto Rico/1934 ${ }^{19}$ flanking the tdKatushka2 reporter sequence and cloned into the dual promoter influenza reverse genetics plasmid using BsmBI restriction sites To prepare the influenza reverse genetics plasmid using chicken beta-actin CAG pol-II promoter (Addgene Cat No. 41583), an insert comprising human pol-I promoter and mouse pol-I terminator sequences in negative orientation flanking two BsmBI restriction sites was cloned using $\mathrm{KpnI}$ and XhoI restriction sites. Then full-length of influenza genes of high-yield A/Puerto Rico/8/1934 ${ }^{77}$ were cloned into BsmBI restriction sites. To prepare plasmids for stable cell line development, sequences of Streptomyces puromycin $N$-acetyl-transferase (PAC), which confers resistance to puromycin, followed by self-cleaving peptide of Thosea asigna virus 2A (T2A) and coding region of influenza A PB1, which has PB1-F2 transcript unmodified, or HA genes were synthesized and cloned into pCAGGS plasmid (Addgene Cat No. 41583) using KpnI and XhoI restriction sites. All plasmids were confirmed by Sanger sequencing.

Cells. To propagate influenza viruses, MDCK-SIAT1 cells (Millipore Sigma) were used. Cells were maintained with complete media comprising Dulbecco's modified Eagle's medium high glucose (DMEM; ThermoFisher) supplemented with $10 \%$ (v/

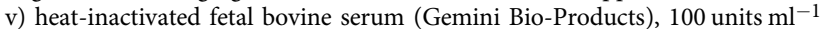
penicillin (ThermoFisher), $100 \mu \mathrm{g} \mathrm{ml}^{-1}$ streptomycin (ThermoFisher), and geneticin $\left(1 \mathrm{mg} \mathrm{ml}^{-1}\right)$ (ThermoFisher). To develop constitutively PB1- or HAexpressing MDCK-SIAT1 cells, one plasmid encoding both puromycin resistance and influenza genes was transfected into MDCK-SIAT1 cells using Lipofectamine 2000 (ThermoFisher). Two days post transfection, cells were transferred from 6well plates into $10-\mathrm{cm}$ dishes containing DMEM media with $10 \%$ bovine serum (Gemini Bio-Products), penicillin, streptomycin, geneticin, and puromycin (0.25 $\mu \mathrm{g} \mathrm{ml}^{-1}$; ThermoFisher) for selection. Medium was changed every $48 \mathrm{~h}$. Clonal selection was performed using 8 or $10 \mathrm{~mm}$ cloning cylinders (Fisher Scientific) about 2 weeks after the transfection. Clonal cell lines were screened using a reporter virus prepared on a polyclonal cell line. To rescue influenza viruses, Flp-In 293 cells (ThermoFisher) were transfected as described below.

Reverse genetics of influenza viruses. For molecular clone influenza viruses, an eight-plasmid approach in which each influenza segment is inserted between pol-II (positive orientation) and pol-I (negative orientation) promoters was used to rescue parental molecular clone viruses. Briefly, Flp-In 293 cells were transfected with dua promoter plasmids encoding each influenza segment of A/WSN/1933 (obtained from St. Jude Children's Research Hospital) and a CMV-driven plasmid expressing human transmembrane serine protease 2 (hTMPRSS2). Transfection was performed using Lipofectamine 3000 in 6-well plates coated with D-lysine. Three days post infection, TPCK-treated trypsin $\left(0.5 \mu \mathrm{g} \mathrm{ml}^{-1}\right.$, Sigma $)$ was added to the transfected cells for 2-4 h. Supernatant was harvested, cleared by centrifugation $(200 \times g, 10 \mathrm{~min})$, and used for propagation in MDCK-SIAT1 cells by limiting dilution. For virus propagation, the inoculum was prepared in virus growth medium comprising of OptiMEM (ThermoFisher) supplemented with TPCKtreated trypsin at $1 \mathrm{\mu g} \mathrm{ml}^{-1}$. MDCK-SIAT1 flasks containing cells at $\sim 80 \%$ confluence were washed twice with phosphate-buffered saline (PBS) and incubated with the inoculum at $37^{\circ} \mathrm{C}$ for virus adsorption. After $1 \mathrm{~h}$, the inoculum was removed, cells were washed with $\mathrm{PBS}$, and virus growth media was added to the cells. The infected cells were incubated at $37^{\circ} \mathrm{C}$ in a humidified $5 \% \mathrm{CO}_{2}$ atmosphere. After 2 days, supernatant was harvested when cytopathic effect reached $40-60 \%$, cleared by centrifugation $(200 \times g, 10 \mathrm{~min})$, aliquoted, and stored at $-80^{\circ}$ C. For influenza HA and NA sequencing, viral RNA was extracted with the RNeasy Extraction Kit (Qiagen). Influenza viral RNA was amplified using the Qiagen OneStep RT-PCR Kit (Qiagen) and HA- and NA-specific primers tagged with M13 sequences. Each amplicon was sequenced with M13 primers in both 
directions. Primer sequences are available upon request. Sequences were analyzed using Sequencer 5.4 (Gene Codes).

For R3 $\triangle \mathrm{PB} 1$ influenza viruses, Flp-In 293 cells were transfected with eight dual promoter influenza reverse genetics plasmids (the PB1 reporter segment plasmid replaced the plasmid encoding PB1 of A/WSN/1933) together with pol-II-driven expression plasmid encoding PB1 of A/WSN/1933 and hTMPRSS2. Rescued viruses were propagated in MDCK-SIAT1 cells constitutively expressing PB1 of A/ WSN/1933 in the presence of TPCK-treated trypsin $\left(1 \mu \mathrm{g} \mathrm{ml}^{-1}\right)$. Virus stocks were stored at $-80^{\circ} \mathrm{C}$.

For replication-restricted rewired $\Delta$ PB1 influenza viruses, Flp-In 293 (ThermoFisher) cells were transfected with eight dual promoter influenza reverse genetics plasmids (PB1 segment comprises PB1 genome packaging signals flanking the coding region of an HA segment in which the HA genome packaging signals have been destroyed by introducing silent mutations, while the HA segment of $\mathrm{R} 4 \triangle \mathrm{PB} 1$ contains the reporter gene flanked by intact HA genomic packaging signals) together with pol-II-driven expression plasmid encoding PB1 of A/WSN/ 1933 and hTMPRSS2. Rescued viruses were propagated as described above.

For R3 $\Delta$ HA influenza genes, Flp-In 293 cells were transfected with eight influenza reverse genetics plasmids encoding sequences of high-yield A/Puerto Rico/8/1936 (HA segment was replaced with HA reporter segment described above), pol-II-driven HA and hTMPRSS2 expressing plasmids. Viruses were propagated in MDCK-SIAT1 cells constitutively expressing influenza HA gene in the presence of TPCK-treated trypsin $\left(1 \mu \mathrm{g} \mathrm{ml}{ }^{-1}\right)$. Virus stocks were stored at $-80^{\circ} \mathrm{C}$

\section{Phylogenetic and evolution-based conservation analyses of influenza HA.} Nucleotide sequences of mature H1 HA $(N=25)$ and H3 HA $(N=26)$ protein were aligned using Muscle algorithm found in Bioedit v7.2.5. Phylogenetic trees were generated using neighbor-joined methods and Kimura 2-parameter substitution model as implemented in MEGA v10. Evolution-based conservation analyses of amino acid residues in the extracellular region of $\mathrm{H} 1$ and $\mathrm{H} 3 \mathrm{HA}$ proteins was done using Consurf (http://consurf.tau.ac.il) and visualized on the atomic structures of HA proteins of A/California/07/2009 (PDB ID: 3LZG) and A/ Victoria/361/2011 (PDB ID: 4WE8). To evaluate the conservation of each amino acid of H3 HA proteins isolated from influenza viruses circulating in humans, fulllength H3 HA sequences of human H3N2 viruses were obtained from the GISAID database (http://platform.gisaid.org). Alignment of nucleotide sequences was performed using MAFFT v7 server-based algorithm using default settings (https:// mafft.cbrc.jp/alignment/server/large.html). After removal of redundant sequences and sequences with gaps or degenerate nucleotide bases, we obtained a dataset of 16,893 unique sequences. Due to the large size of this sequence dataset, we clustered sequences (CD-HIT EST at http://weizhong-lab.ucsd.edu/cdhit-web-server/ cgi-bin/index.cgi? $\mathrm{cmd}=$ cd-hit-est) with identity $>99.6 \%$ and choose a representative sequence from each cluster. The final dataset used to estimate evolution-based conservation of amino acid residues in human H3 HA proteins had 3408 sequences. Similarly, an alignment of $1222 \mathrm{H} 1 \mathrm{HA}$ of H1N1 viruses circulating in humans between 1918 and 2019 sequences was used to evaluate the conservation of each amino acid of this protein.

Virus titration and neutralization. For molecular clones, ELISA-based influenza MN assay was performed following WHO-recommended protocol. Briefly, influenza viruses were titrated in MDCK-SIAT1 cells plated in 96-well plates at 50,000 cells $\mathrm{ml}^{-1} 24 \mathrm{~h}$ before infection. Virus dilutions were prepared using OptiMEM supplemented with TPCK-treated trypsin $\left(1 \mu \mathrm{g} \mathrm{ml}^{-1}\right)$. Infected cells were incubated at $37^{\circ} \mathrm{C}$ in a humidified $5 \% \mathrm{CO}_{2}$ atmosphere. After $18 \mathrm{~h}$, cells were fixed with $80 \%$ cold acetone and air dried. Virus replication was detected by biotinconjugated antibodies to influenza virus nucleoprotein (MAB8257B and MAB8258B, Millipore Sigma) and was visualized with horseradish peroxidaseconjugated streptavidin and SureBlue TMB Microwell Peroxidase Substrate (KPL). Absorbance was read at $450 \mathrm{~nm}$ (A450) and $650 \mathrm{~nm}$ (A650) with the SpectraMax Paradigm microplate reader (Molecular Devices). The A650 was used to subtract plate background. Half-maximal tissue culture infectious dose $\left(\mathrm{TCID}_{50}\right)$ titer was calculated using Reed-Muench method. Neutralization assays were performed using 100-200 TCID $_{50}$ units of virus and 4-fold antibody dilutions made in OptiMEM supplemented with TPCK-treated trypsin. Virus and antibody were mixed in equal volumes and incubated $1 \mathrm{~h}$ at $37^{\circ} \mathrm{C}$ prior to adding to substrate MDCK-SIAT1 cells. Control wells of virus alone (VC) and diluent alone (CC) were included on each plate. Fifty microliters of antibody-virus mixture were then added to wells of pre-washed cells in duplicate and the plates were incubated for 18 $\mathrm{h}$ at $37^{\circ} \mathrm{C}$ in a humidified $5 \% \mathrm{CO}_{2}$ atmosphere. Infected cells were detected as described above. The percent neutralization was calculated by constraining the VC control as $0 \%$ and the CC control as $100 \%$ and plotted against antibody concentration. A curve fit was generated by a four-parameter nonlinear fit model in Prism (GraphPad). The $80 \%\left(\mathrm{IC}_{80}\right.$ ) inhibitory concentrations were obtained from the curve fit for each antibody.

Titer of $\mathrm{R} 3 \Delta \mathrm{PB} 1$ or $\mathrm{R} 3 \Delta \mathrm{HA}$ viruses was measured in PB1-expressing MDCKSIAT1 cells plated in 96-well black plates with transparent bottom (Greiner) at $18 \mathrm{~h}$ post infection and counting fluorescent foci using Celigo Image Cytometer (Nexcelom) with customized red channel to enhance detection of mKate2/ tdKatushka2 reporter (EX 540/80 nm, DIC $593 \mathrm{~nm}$ and EM 593/LP nm). In Celigo operation and analysis software v4.1, Target 1 protocol was used to detect and count fluorescent foci. Titer was expressed as fluorescent foci per ml. For each neutralization reaction, virus dilution that resulted in cca. 1000 (500-4000) fluorescent foci per well at $18 \mathrm{~h}$ post infection was used. Neutralization assays using $\mathrm{R} 3$ viruses performed to compare the fluorescence- and ELISA-based assays were done in 96-well black transparent bottom plates. Cells were plated $24 \mathrm{~h}$ before the experiment. Neutralization reaction was done as described above. R3 influenza neutralization assay was optimized to be performed in 384-well plate format. PB1expressing MDCK-SIAT1 cells were washed twice with PBS, re-suspended in OptiMEM, and plated $2 \mathrm{~h}$ before the assay in 384-well plates at 150,000 cells ml ${ }^{-1}$ $(20 \mu \mathrm{l}$ per well). Twenty-five microliters of each neutralization mixture consisted of $2 \mu \mathrm{g} \mathrm{ml}^{-1}$ TPCK-treated trypsin and equal parts of virus and 4-fold serial dilutions of antibodies were transferred to wells in quadruplicate. Control wells of virus alone (VC) and diluent alone (CC) were included on each plate. Fluorescent foci were counted at $18-24 \mathrm{~h}$ post infection using a Celigo instrument. Neutralization titers were calculated using Prism as described above.

Detailed protocols are provided as Supplementary Notes 1 and 2.

Antibody preparation. Sequences of immunoglobulin heavy and light chains were synthesized and cloned into human IgG1 as previously described ${ }^{49}$. The expression vectors were transiently transfected into Expi293F (ThermoFisher) using ExpiFectamine 293 transfection reagents (ThermoFisher). mAbs were purified using sepharose Protein-A (GE Healthcare) following the manufacturer's instructions.

Negative stain EM. Virus preparations were mixed at a 1:1 ratio with fixative containing $4 \%$ glutaraldehyde and $0.2 \mathrm{M}$ cacodylate buffer, $\mathrm{pH} 7$. A drop of the fixed sample was placed on a carbon-coated, glow-discharged copper grid for about $30 \mathrm{~s}$. The drop was then removed using filter paper, and the grid was washed with three drops of buffer containing $10 \mathrm{mM}$ HEPES, pH 7, and $150 \mathrm{mM} \mathrm{NaCl}$, followed by negative staining with $0.75 \%$ uranyl formate. Imaging was performed using a ThermoFisher Talos F200C electron microscope operated at $200 \mathrm{kV}$ and equipped with a Ceta camera.

Statistics and reproducibility. All statistical analysis was performed using the GraphPad Prism software. Specific tests used to determine statistical significance are indicated in the "Methods" section and corresponding figure legends. Probability values $<0.05$ were considered statistically significant. All the figures are compiled using Inkscape (v1.0.0).

Reporting summary. Further information on research design is available in the Nature Research Reporting Summary linked to this article.

\section{Data availability}

All images and data were generated and analyzed by the authors and will be made available by the corresponding authors (B.S.G. and M.K.) upon reasonable request. Influenza reverse genetics plasmids were obtained from St. Jude Research Hospital thorough an MTA. All sequences corresponding to the influenza HA, NA, and reporter segments used in the present study have been deposited to NCBI Genbank under accession numbers MW298159-MW298274. A reporting summary for this article is available as a Supplementary Information file. Source data are provided with this paper.

Received: 7 July 2020; Accepted: 22 February 2021; Published online: 19 March 2021

\section{References}

1. Harfoot, R. \& Webby, R. J. H5 influenza, a global update. J. Microbiol. 55, 196-203 (2017).

2. Hutchinson, E. C. \& Yamauchi, Y. Understanding influenza. Methods Mol. Biol. 1836, 1-21 (2018)

3. Vijaykrishna, D. et al. The contrasting phylodynamics of human influenza B viruses. Elife 4, e05055 (2015).

4. Zost, S. J., Wu, N. C., Hensley, S. E. \& Wilson, I. A. Immunodominance and antigenic variation of influenza virus hemagglutinin: implications for design of universal vaccine immunogens. J. Infect. Dis. 219, S38-S45, (2019).

5. Kanekiyo, M., Ellis, D. \& King, N. P. New vaccine design and delivery technologies. J. Infect. Dis. 219, S88-S96 (2019).

6. Crank, M. C., Mascola, J. R. \& Graham, B. S. Preparing for the next influenza pandemic: the development of a universal influenza vaccine. J. Infect. Dis. 219, S107-S109 (2019).

7. Crowe, J. E. Antibody determinants of influenza immunity. J. Infect. Dis. 219, S21-S29 (2019).

8. Krammer, F. \& Palese, P. Universal influenza virus vaccines that target the conserved hemagglutinin stalk and conserved sites in the head domain. $J$. Infect. Dis. 219, S62-S67 (2019). 
9. Laurie, K. L. et al. International laboratory comparison of influenza microneutralization assays for $\mathrm{A}(\mathrm{H} 1 \mathrm{~N} 1) \mathrm{pdm} 09, \mathrm{~A}(\mathrm{H} 3 \mathrm{~N} 2)$, and $\mathrm{A}(\mathrm{H} 5 \mathrm{~N} 1)$ influenza viruses by CONSISE. Clin. Vaccin. Immunol. 22, 957-964 (2015).

10. Donis, R. O. Antigenic analyses of highly pathogenic avian influenza a viruses. Curr. Top. Microbiol. Immunol. 385, 403-440 (2014)

11. Wei, X. et al. Antibody neutralization and escape by HIV-1. Nature 422, 307-312 (2003).

12. Pierson, T. C. et al. A rapid and quantitative assay for measuring antibodymediated neutralization of West Nile virus infection. Virology 346, 53-65 (2006).

13. Chen, M. et al. A flow cytometry-based assay to assess RSV-specific neutralizing antibody is reproducible, efficient and accurate. J. Immunol. Methods 362, 180-184 (2010).

14. Yang, Z. Y. et al. Immunization by avian $\mathrm{H} 5$ influenza hemagglutinin mutants with altered receptor binding specificity. Science 317, 825-828 (2007).

15. Carnell, G. W., Ferrara, F., Grehan, K., Thompson, C. P. \& Temperton, N. J. Pseudotype-based neutralization assays for influenza: a systematic analysis. Front. Immunol. 6, 161 (2015).

16. Oh, H. L. et al. An antibody against a novel and conserved epitope in the hemagglutinin 1 subunit neutralizes numerous $\mathrm{H} 5 \mathrm{~N} 1$ influenza viruses. $J$. Virol. 84, 8275-8286 (2010).

17. Breen, M., Nogales, A., Baker, S. F. \& Martinez-Sobrido, L. Replicationcompetent influenza A viruses expressing reporter genes. Viruses 8, 179 (2016).

18. Bloom, J. D., Gong, L. I. \& Baltimore, D. Permissive secondary mutations enable the evolution of influenza oseltamivir resistance. Science 328, 1272-1275 (2010).

19. Martinez-Sobrido, L. et al. Hemagglutinin-pseudotyped green fluorescent protein-expressing influenza viruses for the detection of influenza virus neutralizing antibodies. J. Virol. 84, 2157-2163 (2010).

20. Nogales, A., Baker, S. F., Domm, W. \& Martinez-Sobrido, L. Development and applications of single-cycle infectious influenza A virus (scilAV). Virus Res. 216, 26-40 (2016).

21. Westgeest, K. B. et al. Genomewide analysis of reassortment and evolution of human influenza A(H3N2) viruses circulating between 1968 and 2011. J. Virol. 88, 2844-2857 (2014).

22. Muramoto, Y. et al. Hierarchy among viral RNA (vRNA) segments in their role in vRNA incorporation into influenza A virions. J. Virol. 80, 2318-2325 (2006).

23. Shcherbo, D. et al. Far-red fluorescent tags for protein imaging in living tissues. Biochem. J. 418, 567-574 (2009).

24. Matrosovich, M., Matrosovich, T., Carr, J., Roberts, N. A. \& Klenk, H. D Overexpression of the alpha-2,6-sialyltransferase in MDCK cells increases influenza virus sensitivity to neuraminidase inhibitors. J. Virol. 77, 8418-8425 (2003).

25. Francis, T. Jr. Vaccination against influenza. Bull. World Health Organ. 8, 725-741 (1953).

26. Smith, D. J. et al. Mapping the antigenic and genetic evolution of influenza virus. Science 305, 371-376 (2004)

27. Bedford, T. et al. Integrating influenza antigenic dynamics with molecular evolution. Elife 3, e01914 (2014).

28. Fonville, J. M. et al. Antibody landscapes after influenza virus infection or vaccination. Science 346, 996-1000 (2014).

29. Anderson, C. S., McCall, P. R., Stern, H. A., Yang, H. \& Topham, D. J. Antigenic cartography of $\mathrm{H} 1 \mathrm{~N} 1$ influenza viruses using sequence-based antigenic distance calculation. BMC Bioinformatics 19, 51 (2018).

30. Nelson, M. I. et al. Multiple reassortment events in the evolutionary history of H1N1 influenza A virus since 1918. PLoS Pathog. 4, el000012 (2008).

31. Kilbourne, E. D. et al. The total influenza vaccine failure of 1947 revisited: major intrasubtypic antigenic change can explain failure of vaccine in a postWorld War II epidemic. Proc. Natl Acad. Sci. USA 99, 10748-10752 (2002).

32. Petrie, J. G. et al. Antibodies against the current influenza $A(H 1 N 1)$ vaccine strain do not protect some individuals from infection with contemporary circulating influenza A(H1N1) virus strains. J. Infect. Dis. 214, 1947-1951 (2016).

33. Nelson, M. I. et al. Introductions and evolution of human-origin seasonal influenza a viruses in multinational swine populations. J. Virol. 88, 10110-10119 (2014).

34. Jhung, M. A. et al. Outbreak of variant influenza A(H3N2) virus in the United States. Clin. Infect. Dis. 57, 1703-1712 (2013).

35. Gao, Q. \& Palese, P. Rewiring the RNAs of influenza virus to prevent reassortment. Proc. Natl Acad. Sci. USA 106, 15891-15896 (2009).

36. WHO/OIE/FAO H5N1 Evolution Working Group. Toward a unified nomenclature system for highly pathogenic avian influenza virus (H5N1) Emerg. Infect. Dis. 14, el (2008).

37. Chen, Y. et al. Human infections with the emerging avian influenza A H7N9 virus from wet market poultry: clinical analysis and characterisation of viral genome. Lancet 381, 1916-1925 (2013).
38. Ledgerwood, J. E. et al. Prime-boost interval matters: a randomized phase 1 study to identify the minimum interval necessary to observe the H5 DNA influenza vaccine priming effect. J. Infect. Dis. 208, 418-422 (2013).

39. DeZure, A. D. et al. An avian influenza H7 DNA priming vaccine is safe and immunogenic in a randomized phase I clinical trial. NPJ Vaccines 2, 15 (2017).

40. Stadlbauer, D. et al. Broadly protective human antibodies that target the active site of influenza virus neuraminidase. Science 366, 499-504 (2019).

41. Wan, H. et al. Structural characterization of a protective epitope spanning A (H1N1)pdm09 influenza virus neuraminidase monomers. Nat. Commun. 6, 6114 (2015).

42. McLellan, J. S. et al. Structure-based design of a fusion glycoprotein vaccine for respiratory syncytial virus. Science 342, 592-598 (2013).

43. Ledgerwood, J. E. et al. DNA priming and influenza vaccine immunogenicity: two phase 1 open label randomised clinical trials. Lancet Infect. Dis. 11, 916-924 (2011).

44. Andrews, S. F. et al. Preferential induction of cross-group influenza A hemagglutinin stem-specific memory B cells after H7N9 immunization in humans. Sci. Immunol. 2, eaan2676 (2017).

45. Kanekiyo, M. et al. Mosaic nanoparticle display of diverse influenza virus hemagglutinins elicits broad B cell responses. Nat. Immunol. 20, 362-372 (2019).

46. Throsby, M. et al. Heterosubtypic neutralizing monoclonal antibodies crossprotective against $\mathrm{H} 5 \mathrm{~N} 1$ and $\mathrm{H} 1 \mathrm{~N} 1$ recovered from human $\mathrm{IgM}+$ memory B cells. PLoS ONE 3, e3942 (2008).

47. Corti, D. et al. A neutralizing antibody selected from plasma cells that binds to group 1 and group 2 influenza A hemagglutinins. Science 333, 850-856 (2011).

48. Ekiert, D. C. et al. A highly conserved neutralizing epitope on group 2 influenza A viruses. Science 333, 843-850 (2011).

49. Whittle, J. R. et al. Broadly neutralizing human antibody that recognizes the receptor-binding pocket of influenza virus hemagglutinin. Proc. Natl Acad. Sci. USA 108, 14216-14221 (2011).

50. Iba, Y. et al. Conserved neutralizing epitope at globular head of hemagglutinin in H3N2 influenza viruses. J. Virol. 88, 7130-7144 (2014).

51. Lee, P. S. et al. Receptor mimicry by antibody F045-092 facilitates universal binding to the $\mathrm{H} 3$ subtype of influenza virus. Nat. Commun. 5, 3614 (2014).

52. Kallewaard, N. L. et al. Structure and function analysis of an antibody recognizing all influenza A subtypes. Cell 166, 596-608 (2016).

53. Krause, J. C. et al. A broadly neutralizing human monoclonal antibody that recognizes a conserved, novel epitope on the globular head of the influenza H1N1 virus hemagglutinin. J. Virol. 85, 10905-10908 (2011).

54. $\mathrm{Wu}, \mathrm{Y}$. et al. A potent broad-spectrum protective human monoclonal antibody crosslinking two haemagglutinin monomers of influenza A virus. Nat. Commun. 6, 7708 (2015).

55. Ekiert, D. C. et al. Cross-neutralization of influenza A viruses mediated by a single antibody loop. Nature 489, 526-532 (2012).

56. Whittle, J. R. et al. Flow cytometry reveals that $\mathrm{H} 5 \mathrm{~N} 1$ vaccination elicits crossreactive stem-directed antibodies from multiple Ig heavy-chain lineages. J. Virol. 88, 4047-4057 (2014).

57. Joyce, M. G. et al. Vaccine-induced antibodies that neutralize group 1 and group 2 influenza A viruses. Cell 166, 609-623 (2016).

58. Hobson, D., Curry, R. L., Beare, A. S. \& Ward-Gardner, A. The role of serum haemagglutination-inhibiting antibody in protection against challenge infection with influenza A2 and B viruses. J. Hyg. 70, 767-777 (1972)

59. Meiklejohn, G., Kempe, C. H., Thalman, W. G. \& Lennette, E. H. Evaluation of monovalent influenza vaccines. II. Observations during an influenza aprime epidemic. Am. J. Hyg. 55, 12-21 (1952)

60. de Jong, J. C. et al. Haemagglutination-inhibiting antibody to influenza virus. Dev. Biol. 115, 63-73 (2003).

61. Coudeville, L. et al. Relationship between haemagglutination-inhibiting antibody titres and clinical protection against influenza: development and application of a Bayesian random-effects model. BMC Med. Res. Methodol. 10, 18 (2010).

62. Jahiel, R. I. \& Kilbourn, E. D. Reduction in plaque size and reduction in plaque number as differing indices of influenza virus-antibody reactions. J. Bacteriol. 92, 1521-\& (1966).

63. Rowe, T. et al. Detection of antibody to avian influenza A (H5N1) virus in human serum by using a combination of serologic assays. J. Clin. Microbiol. 37, 937-943 (1999).

64. Flandorfer, A., Garcia-Sastre, A., Basler, C. F. \& Palese, P. Chimeric influenza A viruses with a functional influenza B virus neuraminidase or hemagglutinin. J. Virol. 77, 9116-9123 (2003).

65. Horimoto, T., Iwatsuki-Horimoto, K., Hatta, M. \& Kawaoka, Y. Influenza A viruses possessing type $B$ hemagglutinin and neuraminidase: potential as vaccine components. Microbes Infect. 6, 579-583 (2004).

66. Ping, J., Lopes, T. J., Neumann, G. \& Kawaoka, Y. Development of high-yield influenza B virus vaccine viruses. Proc. Natl Acad. Sci. USA 113, E8296-E8305 (2016). 
67. Boyoglu-Barnum, S. et al. Quadrivalent influenza nanoparticle vaccines induce broad protection. Nature https://doi.org/10.1038/s41586-021-03365-x (2021). In Press.

68. Lin, Y., Gu, Y. \& McCauley, J. W. Optimization of a quantitative microneutralization assay. J. Vis. Exp. https://doi.org/10.3791/54897 (2016).

69. van Baalen, C. A. et al. ViroSpot microneutralization assay for antigenic characterization of human influenza viruses. Vaccine 35, 46-52 (2017).

70. Jorquera, P. A. et al. Insights into the antigenic advancement of influenza A (H3N2) viruses, 2011-2018. Sci. Rep. 9, 2676 (2019).

71. Naito, T. et al. Generation of a genetically stable high-fidelity influenza vaccine strain. J. Virol. https://doi.org/10.1128/JVI.01073-16 (2017).

72. Georgiev, I. S. et al. Delineating antibody recognition in polyclonal sera from patterns of HIV-1 isolate neutralization. Science 340, 751-756 (2013).

73. Yassine, H. M. et al. Hemagglutinin-stem nanoparticles generate heterosubtypic influenza protection. Nat. Med. 21, 1065-1070 (2015).

74. Corbett, K. S. et al. Design of nanoparticulate group 2 influenza virus hemagglutinin stem antigens that activate unmutated ancestor B cell receptors of broadly neutralizing antibody lineages. MBio https://doi.org/10.1128/ mBio.02810-18 (2019).

75. Impagliazzo, A. et al. A stable trimeric influenza hemagglutinin stem as a broadly protective immunogen. Science 349, 1301-1306 (2015).

76. Hoffmann, E., Neumann, G., Kawaoka, Y., Hobom, G. \& Webster, R. G. A DNA transfection system for generation of influenza A virus from eight plasmids. Proc. Natl Acad. Sci. USA 97, 6108-6113 (2000).

77. Ping, J. et al. Development of high-yield influenza A virus vaccine viruses. Nat. Commun. 6, 8148 (2015).

\section{Acknowledgements}

We thank Richard Webby (St. Jude Research Hospital) for influenza reverse genetics plasmids, Jesse Bloom (Fred Hutchinson Cancer Research Center) for HEK-293 cells expressing PB1 of A/WSN/1933, and Jeffrey Boyington (VRC) for help with sequence and structural analyses. This work was supported by the Intramural Research Program of the Vaccine Research Center, National Institute of Allergy and Infectious Diseases, National Institutes of Health. Electron microscopic data collection and analyses were funded by federal funds from the Frederick National Laboratory for Cancer Research, National Institutes of Health, under contract number HHSN261200800001E, and by Leidos Biomedical Research, Inc. (T.S and Y.T.).

\section{Author contributions}

Conceptualization: A.C., M.K., B.S.G.; methodology: A.C.; formal analysis: A.C., M.K.; investigation: A.C., R.A.G., B.E.F., S.F.A., J.L., C.Y., L.H., T.S., Y.T.; resources: J.E.L.; writing—original draft: A.C., M.K.; writing—review and editing: A.C., S.F.A., J.R.M., B.S.G., M.K.; supervision: A.B.M., J.R.M., B.S.G., M.K.; project administration: M.C.C.; funding acquisition: J.R.M., B.S.G.

\section{Funding}

Open Access funding provided by the National Institutes of Health (NIH).

\section{Competing interests}

The authors declare no competing interests.

\section{Additional information}

Supplementary information The online version contains supplementary material available at https://doi.org/10.1038/s41467-021-21954-2.

Correspondence and requests for materials should be addressed to B.S.G. or M.K.

Peer review information Nature Communications thanks Nicholas Wu and the other anonymous reviewer(s) for their contribution to the peer review of this work.

Reprints and permission information is available at http://www.nature.com/reprints

Publisher's note Springer Nature remains neutral with regard to jurisdictional claims in published maps and institutional affiliations.

Open Access This article is licensed under a Creative Commons Attribution 4.0 International License, which permits use, sharing, adaptation, distribution and reproduction in any medium or format, as long as you give appropriate credit to the original author(s) and the source, provide a link to the Creative Commons license, and indicate if changes were made. The images or other third party material in this article are included in the article's Creative Commons license, unless indicated otherwise in a credit line to the material. If material is not included in the article's Creative Commons license and your intended use is not permitted by statutory regulation or exceeds the permitted use, you will need to obtain permission directly from the copyright holder. To view a copy of this license, visit http://creativecommons.org/ licenses/by/4.0/.

This is a U.S. government work and not under copyright protection in the U.S.; foreign copyright protection may apply 2021 